\title{
Estudio de inserción laboral de los graduados, carrera Marketing, Universidad Técnica Estatal de Quevedo, con perspectiva al desarrollo socioeconómico regional y nacional, año 2018 \\ Study of labor insertion of graduates, Marketing career, State Technical University of Quevedo, with a perspective on regional and national socioeconomic development, year 2018
}

\author{
Ing. Jenny Maldonado Castro ${ }^{1}$ \\ jmaldonado@uteq.edu.ec \\ Ing. Diana Isabel Cadena Miranda ${ }^{1}$ \\ dcadena@uteq.edu.ec \\ Ing. María del Carmen Guzmán Macías ${ }^{1}$ \\ mguzman@uteq.edu.ec \\ Eco. Galo Henry Macías España ${ }^{1}$ \\ gmacias@uteq.edu.ec
}

Recibido: 1/07/2018, Aceptado: 1/09/2018

\begin{abstract}
RESUMEN
Los cambios galopantes en todos los niveles y aristas, que se dan en un mundo cada vez más globalizado, obligan implícita y explícitamente a generar profesionales que deben desenvolverse en un ambiente altamente competitivo. La academia, y en particular la educación superior tienen la tarea de ofertar a la sociedad, profesionales competitivos, éticos, dinámicos, holísticos y humanistas. En correspondencia con aquello, la Universidad Técnica Estatal de Quevedo (U.T.E.Q.), tiene entre sus indicadores de evaluación, actividades de seguimiento a los estudiantes graduados de sus aulas. El presente trabajo describe a través de un estudio muestral y de campo, la realidad laboral de los estudiantes graduados en la carrera Marketing de la U.T.E.Q., durante los años 2006 hasta el 2017, en el cual se pretende evidenciar los aspectos positivos, negativos y sujetos a mejoras que desde la academia se puede tributar para el progreso en las condiciones socioeconómicas de los estudiantes y la sociedad en general. La revolución digital y el apogeo de los servicios requieren evolucionar respecto los modelos clásicos del marketing que en muchos ambientes siguen dominando.
\end{abstract}

\footnotetext{
1 Docente Universidad Técnica Estatal de Quevedo Unidad, Facultad de Ciencias Empresariales. Ecuador
} 
Palabras claves: graduados, marketing, empleabilidad

\begin{abstract}
The galloping changes at all levels and edges, which occur in an increasingly globalized world, implicitly and explicitly require professionals to develop in a highly competitive environment. The academy and in particular higher education have the task of offering society, competitive, ethical, dynamic, holistic and humanistic professionals. In correspondence with that, the State Technical University of Quevedo (U.T.E.Q.), has among its evaluation indicators, follow-up activities to graduate students in their classrooms. The present work describes through a sample and field study, the labor reality of the graduated students in the Marketing career of the UTEQ, during the years 2006 until the 2017, in which it is tried to show the positive, negative aspects and subjects to improvements that from the academy can be taxed for the progress in the socioeconomic conditions of students and society in general.
\end{abstract}

Keywords: graduates, marketing, employability

\title{
Introducción
}

La carrera marketing, mediante la formación de los profesionales, debe fomentar y promover la integración económica que permita desarrollar capacidades propias y reducir la dependencia económica del exterior, para fortalecer el comercio intrarregional y la creación de cadenas de valor que impulsen la producción con valor agregado a los productos y garantizar mercados de consumo. Las ciencias de la administración, económicas y de la gestión, donde se enmarca al marketing, permiten evidenciar cómo el mundo del marketing se encuentra sujeto a un entorno dinámico y de cambios constantes, y lo que en un momento podría ser la fórmula del éxito, en poco tiempo caduca y frena su desarrollo, por lo cual es imperante y cada vez más fuerte la responsabilidad de la academia en generar profesionales capaces y alta marketing mente competitivos, pero además se necesita de procesos que extrapolen la responsabilidad de las aulas hasta el desempeño de dichos profesionales en su ejercicio profesional.

El Sistema de Educación Superior del Ecuador, enfrenta el reto de articular su oferta académica con las necesidades contempladas en los planes de desarrollo nacional, provincial y local, para contribuir al desarrollo del contexto social, tal como lo dispone el criterio de pertinencia contemplado en el artículo 107 de la Ley Orgánica de Educación Superior (LOES).

Desde la perspectiva interna, la visión de la UTEQ hasta el 2020 precisa: "La Universidad Técnica Estatal de Quevedo ocupa los primeros lugares entre las instituciones de Educación Superior ecuatorianas por su calidad académica, investigativa y de servicios que contribuyen al Buen Vivir". Concomitante con ello, su misión señala: "Formar profesionales y académicos con visión científica y humanista capaces de desarrollar investigaciones, crear tecnologías, mantener y difundir 




No. 20, 31 de octubre de 2018

ISSN impreso: 1390 - 6321

nuestros saberes y culturas ancestrales, para la construcción de soluciones a los problemas de la región y el país".

Según Kotler y Keller (2006), el intercambio de bienes y servicios incluye cuatro aspectos fundamentales: ¿cuándo?, ¿dónde?, ¿a quién? y ¿cómo?

En el primero, los autores se refieren al momento preciso de llevarlo a efecto; en el segundo, a la estrategia geográfica; el tercero, a la definición del público objetivo y finalmente, se hace una referencia a la estrategia a seguir.

Para contribuir al Comercio y a la Matriz Productiva, la carrera de Marketing de la Universidad Técnica Estatal de Quevedo formará profesionales que apliquen conocimientos teóricos y prácticos en la formulación de planes de mercadotecnia y de negocios, para potenciar los emprendimientos y la innovación en el desarrollo de nuevos productos y servicios para dar solución a los problemas del contexto nacional, regional y global.

La carrera de Marketing de la Universidad Técnica Estatal de Quevedo, formará profesionales estrategas, con gestión administrativa, emprendedora e innovadora, identificando su entorno y potenciándolo comercialmente con valor agregado, sustentado en el Plan Nacional de Buen Vivir (Secretaría Nacional de Planificación y Desarrollo, 2013) y que interactúan con el objeto de esta profesión en el contexto: Economía Social y Solidaria.

Como lo señalan Arcaide, Bernúez, Díaz-Aroca, Expinosa y Muñis (2013), muchos empresarios, sobre todo los micro y pequeños, desconocen todas las actividades que involucra el marketing y por ende el papel tan relevante que desempeña ésta en el éxito empresarial. No se debe olvidar que tal vez no todas las empresas sean globales, pero sí todas tendrán que enfrentar a competidores globales (Castillo, Bohórquez y Pérez, 2013).

Los retos que generan la revolución digital y el auge de los servicios exigen evolucionar respecto los modelos clásicos del marketing que en muchos entornos siguen imperando (Albornoz, 2009). Las ciencias de la administración, económicas y de la gestión donde podemos enmarcar al marketing permiten sustentar teóricamente la presencia del paradigma de la complejidad, evidenciando cómo el mundo del marketing se encuentra sujeto a un entorno similar, dinámico y de cambios constantes. Modelos como las $4 \mathrm{P}$ de Marketing, que datan de los años 60, o el concepto publicitario AIDA que data de 1898, necesitan ser reemplazados por nuevos paradigmas, que entiendan las exigencias de un nuevo consumidor y capturen las oportunidades de la digitalización (Castell, 2003). Por lo que los mercadólogos y sus organizaciones sobrevivirán en la nueva era, enfrentándose de manera exitosa al mercado globalizado, pero para ello deben ser portadores conscientes en su modo de pensar y actuar si saben planificarse, actuar y tener la capacidad para identificar el mercado potencial y sus clientes efectivos (Morales, 
2014).

Sin duda, el capital humano constituye el recurso más estratégico, y también el más complejo de gestionar. El capital humano, esto es, el conjunto de habilidades, conocimientos y competencias de las personas que trabajan en la empresa, en una fuente incuestionable de ventajas competitivas a largo plazo (Blanco, Prado y Mercado, 2016).

\section{Métodos}

La carrera de marketing tiene hasta la fecha una cantidad de 577 graduados, para lo que se optó por determinar una muestra de la población antes indicada.

Población de estudio:

Para ello se partió con la definición de la fórmula a aplicar, la cual se observa abajo. Se estableció un margen de error estadístico de $+/ 10 \%$. El valor del nivel de confianza

$$
n=\frac{\mathrm{s}^{2} \mathrm{pqN}}{\mathrm{E}^{2}(N-1)+\mathrm{s}^{2} \mathrm{pq}}
$$

" $s$ ", se obtuvo de la tabla de la distribución normal, para el valor de $\delta$ $=90 \%$ es 1,65 . Los valores de " $p$ " $y$ " $q$ " son las probabilidades, a priori, de la respuesta binomial, usualmente se toman $p=q=0,5$. Siendo $q=1-p$.

Lo expuesto permite calcular la muestra de la siguiente manera:

\section{Datos}

$\mathrm{N}=577$

$E=10 \%$

$\delta=90 \%$ (margen de confianza) $s=1.96$

$\mathrm{p}=0,5$

$q=0,5$

Si despejamos $\mathrm{n}$ de la fórmula anterior, entonces:

$\mathrm{n}=61$

\section{Resultados}

Luego de la aplicación de la encuesta y del procesamiento de datos respectivo, se pudieron obtener los siguientes resultados. 


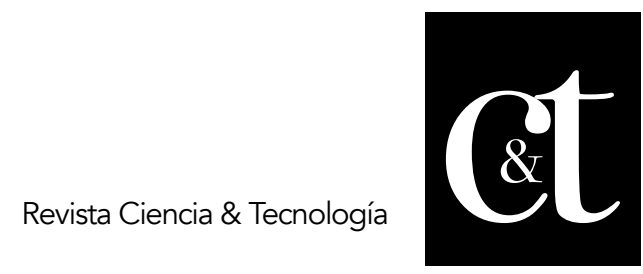

No. 20, 31 de octubre de 2018

ISSN impreso: 1390 - 6321



Gráfico 1. Datos e información personal del graduado género

Fuente: Informe de Seguimiento a Graduados Segundo Período Académico 2017-2018 Carrera Marketing U.T.E.Q.

El $67 \%$ de los graduados que se encuestaron, son de género femenino, en contraparte de un $33 \%$ de género masculino, este mayor número de mujeres graduadas de la carrera, es consecuente con los datos del universo de graduados, que en su mayor porcentaje son mujeres y refleja un aspecto positivo en función de propender cada vez más a la equidad de género.



\section{Gráfico 2. Estado civil actual}

Fuente: Informe de Seguimiento a Graduados Segundo Período Académico 2017-2018 Carrera Marketing U.T.E.Q.

El mayor porcentaje que corresponde al $70 \%$ de los graduados se encuentra actualmente solteros, seguido por un $15 \%$ que están casados, un $13 \%$ en unión libre, y un $2 \%$ tiene como estado civil divorciado. 


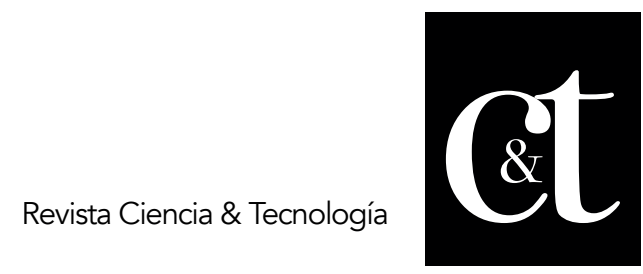

No. 20, 31 de octubre de 2018

ISSN impreso: 1390 - 6321

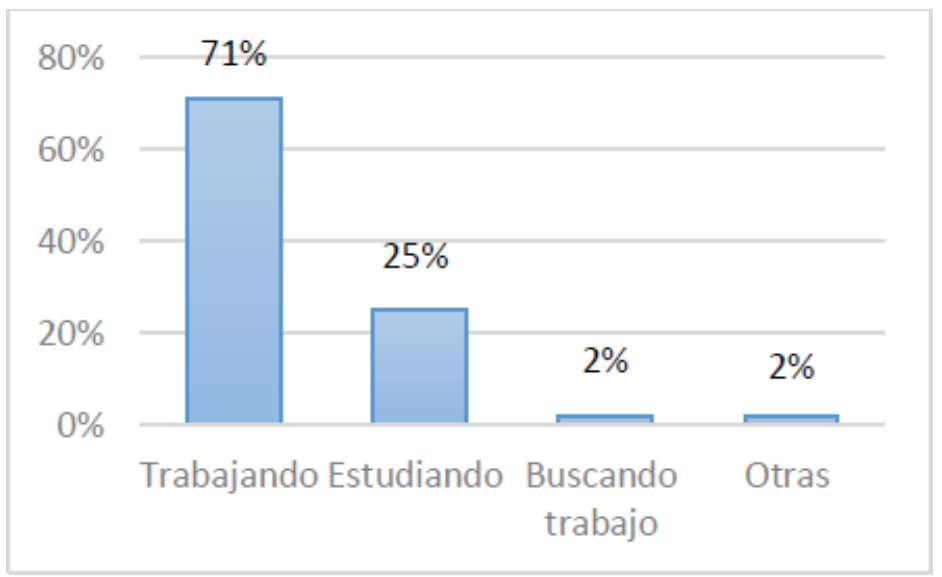

Gráfico 3. Empleabilidad

En la actualidad, ¿en qué actividad ocupa la mayor parte de su tiempo?

Fuente: Informe de Seguimiento a Graduados Segundo Período Académico 2017-2018

Carrera Marketing U.T.E.Q.

El $71 \%$ de los encuestados ocupa la mayor parte de su tiempo trabajando, el $25 \%$ se encuentra estudiando un $2 \%$ está buscando un trabajo, y otro $2 \%$ está realizando alguna otra actividad.

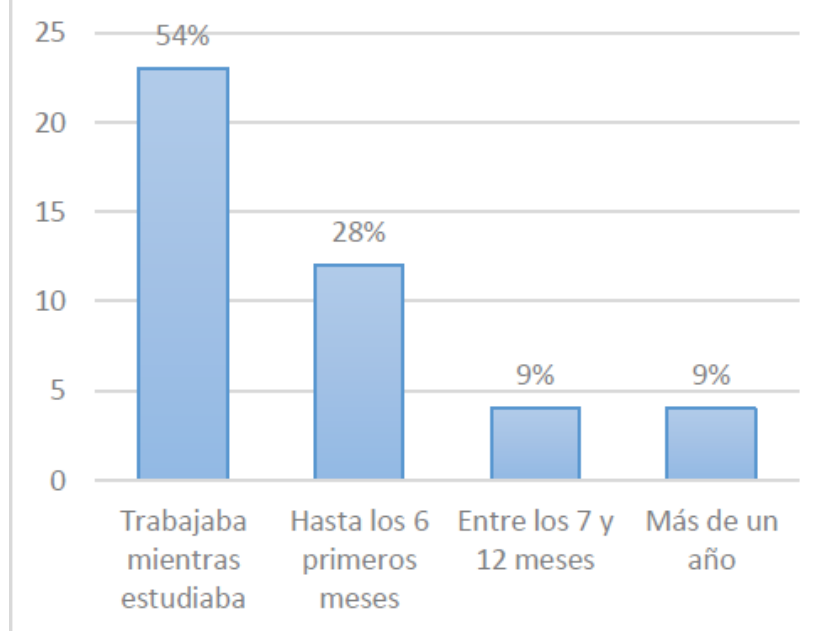

\section{Gráfico 4. ¿Cuánto tiempo después de su graduación le tomó conseguir su primer empleo?}

Fuente: Informe de Seguimiento a Graduados Segundo Período Académico 2017-2018 Carrera Marketing U.T.E.Q.

Maldonado, Cadena, Guzmán, Macías. Estudio de inserción laboral de los graduados, Carrera Marketing, Universidad Técnica Estatal de Quevedo, con perspectiva al desarrollo socioeconómico regional y nacional, año 2018 
El $54 \%$ de los encuestados trabajaba mientras estudiaba, seguido por un $28 \%$ a quienes les tomó 6 meses conseguir su primer trabajo luego de graduarse, porcentajes menores del $9 \%$ cada uno se encuentran en los encuestados que les tomó entre 7 meses y más de un año conseguir su primer empleo luego de haberse graduado.

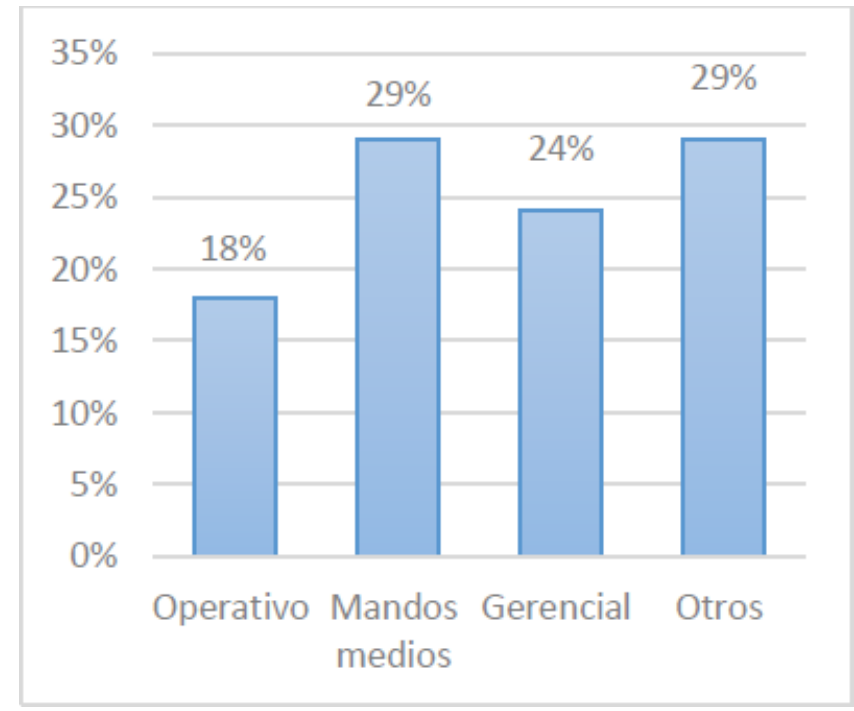

\section{Gráfico 5. ¿En qué cargo se desempeña usted?}

Fuente: Informe de Seguimiento a Graduados Segundo Período Académico 2017-2018 Carrera Marketing U.T.E.Q.

El $29 \%$ de los graduados encuestados ocupa cargos de mandos medios, en las empresas donde laboran, existe otro $29 \%$ que tiene ocupaciones eventuales o está desempleado, el $24 \%$ ocupa cargos gerenciales, y un $18 \%$ tiene cargos operativos.

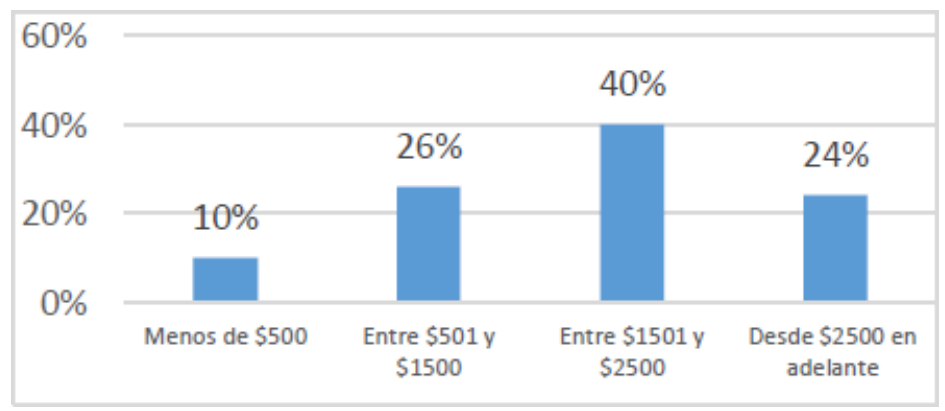

Gráfico 6. ¿Cuál es su ingreso mensual por su actividad profesional?

Fuente: Informe de Seguimiento a Graduados Segundo Período Académico 2017-2018 Carrera Marketing U.T.E.Q. 
De la población de graduados encuestados un $40 \%$ tiene un ingreso mensual entre $\$ 1501$ a $\$ 2500$, un $26 \%$ gana entre $\$ 501$ a $\$ 1500$, el $24 \%$ gana de $\$ 2500$ en adelante, y un $10 \%$ gana menos de $\$ 500$.

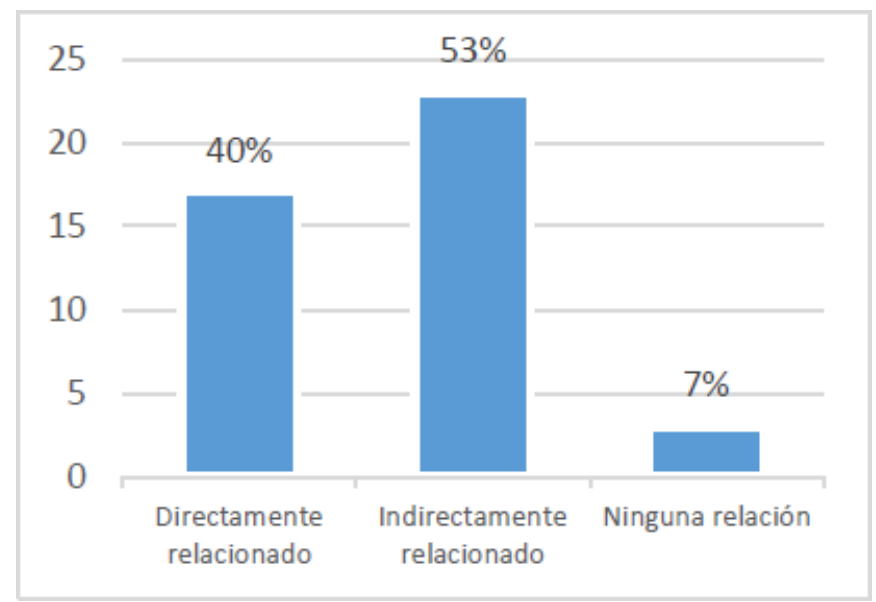

\section{Gráfico 7. ¿En qué medida está relacionado su trabajo actual con su preparación profesional?}

Fuente: Informe de Seguimiento a Graduados Segundo Período Académico 2017-2018 Carrera Marketing U.T.E.Q.

El $53 \%$ de los encuestados indicó que su trabajo actual está indirectamente relacionado con su formación profesional, el $40 \%$ manifestó que está directamente relacionado y un menor porcentaje del $7 \%$ expresó que su trabajo actual no mantiene ninguna relación con su trabajo actual.

\section{Discusión}

De acuerdo a la información registrada, el $67 \%$ de los encuestados que se graduaron de la carrera marketing son de género femenino y un $33 \%$ masculino, lo cual refleja un alto nivel de oportunidad de mejora en la calidad de vida de las mujeres, aportando a la equidad de género y disminución de las brechas de desigualdad respecto a este fenómeno social.

El $71 \%$ de la población encuestada se encuentra laborando, un $25 \%$ está estudiando, un $2 \%$ no realiza ninguna actividad y el otro $2 \%$ tiene otras actividades, estas cifras son un reflejo positivo de los niveles de empleabilidad, aunque con algunas oportunidades de mejora, en cuanto a los tipos de cargo que desempeñan los graduados, como se verá más adelante.

Del total de los encuestados que se encuentran actualmente trabajando, el $29 \%$ ocupa cargos de mandos medios en las empresas donde laboran, otro porcentaje igual del $29 \%$ corresponde a "otros", entre los cuales están los que no tienen trabajo o realizan trabajos eventuales, el $24 \%$ tiene cargos gerenciales, y un $18 \%$ ocupa 
cargos operativos, es importante reconocer en este aspecto que hace falta generar procesos de vinculación e integración, que permitan forjar mayores oportunidades de ocupar cargos gerenciales, más estratégicos y menos operativos, lo cual se transforma en mayores ingresos y en puestos más relacionados con su perfil profesional.

De la población de graduados encuestados un $40 \%$ tiene un ingreso mensual entre $\$ 1501$ a $\$ 2500$, lo cual es bastante aceptable en el contexto económico nacional, un $26 \%$ gana entre $\$ 501$ a $\$ 1500$, que se mantiene en los rangos plausibles, un $24 \%$ gana de $\$ 2500$ en adelante, lo cual representa un porcentaje importante para este valor y un $10 \%$ gana menos de $\$ 500$.

\section{Conclusión}

En síntesis, la inserción laboral de los graduados de la carrera marketing de la UTEQ tiene un porcentaje aceptable e importante, sin que aquello soslaye la necesidad de trabajar en generar mejores oportunidades, desde su formación académica, así como en la vinculación con la sociedad para facilitar oportunidades de ocupar espacios laborales acordes a su perfil profesional y con mejores remuneraciones de sueldos.

Queda la tarea pendiente desde la academia y la UTEQ, en especial, de tributar profesionales que sean entes transformadores de su entorno y no solo agentes de recepción de las condiciones dadas en el contexto.

\section{Referencias bibliográficas}

Albornoz, M. (2009). Indicadores de innovación: las dificultades de un concepto en evolución. Revista CTS, 9-25.

Arcaide, J., Bernúez, S., Díaz-Aroca, E., Expinosa, R. y Muñis, R. (2013). Marketing y pymes. Las principales claves de marketing en la pequeña y mediana empresa. Madrid

Blanco, A., Prado, A. y Mercado, C. (2016). Introducción al marketing y la comunicación de la empresa (Segunda ed.). Madrid, España: Esic.

Castell, J. V. (2003). Tecnología e innovación en la empresa. Catalunya: Universidad Politécnica de Catalunya.

Castillo, V., Bohórquez, M. y Pérez, A. (2013). La mercadotecnia, factor clave para la competitividad organizacional. Gestión Social, 15-30.

Kotler, P., y Keller, K. (2006). Dirección de Marketing (Duodécima ed.). México, México: Pearson Educación.

Morales, D. V. (2014). Innovación y marketing de servicios en la era digital. Madrid, España: ESIC 




No. 20, 31 de octubre de 2018

ISSN impreso: 1390 - 6321

Secretaría Nacional de Planificación y Desarrollo (2013). Plan Nacional para el Buen Vivir. Plan Nacional para el Buen Vivir. Quito, Pichincha, Ecuador: SENPLADES.

Maldonado, Cadena, Guzmán, Macías. Estudio de inserción laboral de los graduados, Carrera Marketing, Universidad Técnica Estatal de Quevedo, con perspectiva al desarrollo socioeconómico regional y nacional, año 2018 